\title{
The Dangers of Immediately Performing a Sensitive and Specific Investigation versus the Dangers of Delaying It: A Cautionary Case of Hodgkin's Lymphoma
}

\author{
Takahiro Ito, Hiroshi Sawachika, Yukinori Harada, Taro Shimizu \\ Department of Diagnostic and Generalist Medicine, Dokkyo Medical University Hospital, Mibu, Tochigi, Japan
}

\section{Doi: 10.12890/2020_002138 - European Journal of Case Reports in Internal Medicine - ๑ EFIM 2020}

Received: 08/11/2020

Accepted: $14 / 11 / 2020$

Published: 01/12/2020 How to cite this article: Ito T, Sawachika H, Harada Y, Shimizu T. The dangers of immediately performing a sensite and specific investigation versus the
dangers of delaying it: a cautionary case of Hodgkin's lymphoma. EJCRIM 2020;7: doi:10.12890/2020_002138.

Conflicts of Interests: The Authors declare that there are no competing interests.

This article is licensed under a Commons Attribution Non-Commercial 4.0 License

\section{ABSTRACT}

A 60-year-old man was admitted with a 1-month history of fever and weight loss. Multiple lymphadenopathies and haemophagocytic lymphohistiocytosis were noted from the beginning, suggesting lymphoma. However, lymph node biopsy was deferred because lymph node biopsy was regarded as being invasive and requires general anaesthesia, and because other possible differential diagnoses including gastrointestinal malignancies and TAFRO syndrome were being considered. Instead, investigations including gastrointestinal endoscopy and bone marrow biopsy were prioritized. The patient was eventually diagnosed with Hodgkin's lymphoma based on lymph node biopsy but died during chemotherapy. Physicians should prioritize the tests that are most directly related to the diagnostic outcome, even if they are invasive.

\section{LEARNING POINTS}

- Lymph node biopsy should be planned early in the course of investigation in patients with enlarged supraclavicular lymph nodes for the diagnosis of malignant lymphoma.

- Hodgkin's lymphoma can be associated with hemophagocytic lymphohistiocytosis and myelofibrosis.

- Analytical diagnostic reasoning based on the evidence is vital for timely and appropriate diagnosis in patients with uncommon presentations.

\section{KEYWORDS}

Hodgkin's lymphoma, myelofibrosis, TAFRO syndrome, diagnostic error

\section{CASE DESCRIPTION}

A 60-year-old man with uncontrolled type 2 diabetes (HbA1c 8.0\%) visited our hospital with a 1-month history of fever, general malaise, loss of appetite, and an approximately $20 \mathrm{~kg}$ weight loss. On admission, high grade fever $\left(39.5^{\circ} \mathrm{C}\right)$, hypotension and tachypnoea were noted, and blood tests showed elevated creatinine, lactate dehydrogenase and C-reactive protein levels, and coagulation abnormalities (Table 1). Chest and abdominal computed tomography (CT) without contrast revealed multiple mild lymphadenopathies on the left supraclavicular fossa and para-abdominal aorta. The patient was initially treated with broad-spectrum antibiotics as sepsis was suspected based on his acute fever with systemic signs. However, blood and urine cultures on admission were sterile, and the patient's condition did not improve. Moreover, pancytopenia developed from day 3 (Table 1). In a search for a possible source of infection, contrast-enhanced chest and abdominal CT 
was performed on day 6, which revealed newly developed splenomegaly, slight gastric wall thickening, a partially narrowed sigmoid colon, increased density of the marrow of both iliac bones, and lymphadenopathies (Fig. 1). Serum ferritin and soluble interleukin 2 receptor (sIL$2 \mathrm{R}$ ) levels were $3,129 \mathrm{ng} / \mathrm{ml}$ and $16,300 \mathrm{U} / \mathrm{ml}$, respectively.

\begin{tabular}{|l|l|l|l|l|l|l|}
\hline & & Day 1 & Day 3 & Day 10 & Day 20 & Day 28 \\
\hline White blood cell count & $\times 10^{3} / \mathrm{mm}^{3}$ & 5.3 & 2.9 & 2.8 & 2.7 & 11.4 \\
\hline Haemoglobin & $\mathrm{g} / \mathrm{dl}$ & 9.7 & 8.1 & 6.9 & 6.5 & 7.3 \\
\hline Platelet count & $\times 10^{4} / \mathrm{mm}^{3}$ & 20.5 & 13.5 & 4.9 & 3.8 & 1.4 \\
\hline Albumin & $\mathrm{U} / \mathrm{I}$ & 2.8 & & 1.6 & & 1.8 \\
\hline $\begin{array}{l}\text { Aspartate } \\
\text { aminotransferase }\end{array}$ & $\mathrm{U} / \mathrm{l}$ & 13 & 15 & 14 & 15 & 47 \\
\hline $\begin{array}{l}\text { Alanine } \\
\text { aminotransferase }\end{array}$ & $\mathrm{U} / \mathrm{l}$ & 23 & 23 & 30 & 21 & 35 \\
\hline Lactate dehydrogenase & $\mathrm{U} / \mathrm{l}$ & 330 & 247 & 300 & 343 & 569 \\
\hline Alkaline phosphatase & $\mathrm{U} / \mathrm{I}$ & & & & 1136 & 806 \\
\hline Total bilirubin & $\mathrm{mg} / \mathrm{dl}$ & 1.6 & 1.9 & 2.1 & 6.4 & 19.7 \\
\hline Direct bilirubin & $\mathrm{mg} / \mathrm{dl}$ & 1.0 & 1.2 & 1.6 & 5.1 & \\
\hline Blood urea nitrogen & $\mathrm{mg} / \mathrm{dl}$ & 103 & 67 & 20 & 36 & 109 \\
\hline Creatinine & $\mathrm{mg} / \mathrm{dl}$ & 3.35 & 1.54 & 0.46 & 0.77 & 2.17 \\
\hline C-reactive protein & $\mathrm{mg} / \mathrm{dl}$ & 12.81 & 12.83 & 14.01 & 14.93 & 13.88 \\
\hline Prothrombin time & $\mathrm{sec}$ & 20.7 & 20.7 & 16.8 & & 27.5 \\
\hline Partial thrombin time & $\mathrm{sec}$ & 50.9 & 51.1 & 46.9 & & 61.0 \\
\hline Fibrinogen & $\mathrm{mg} / \mathrm{dl}$ & 321 & & 321 & & 264 \\
\hline
\end{tabular}

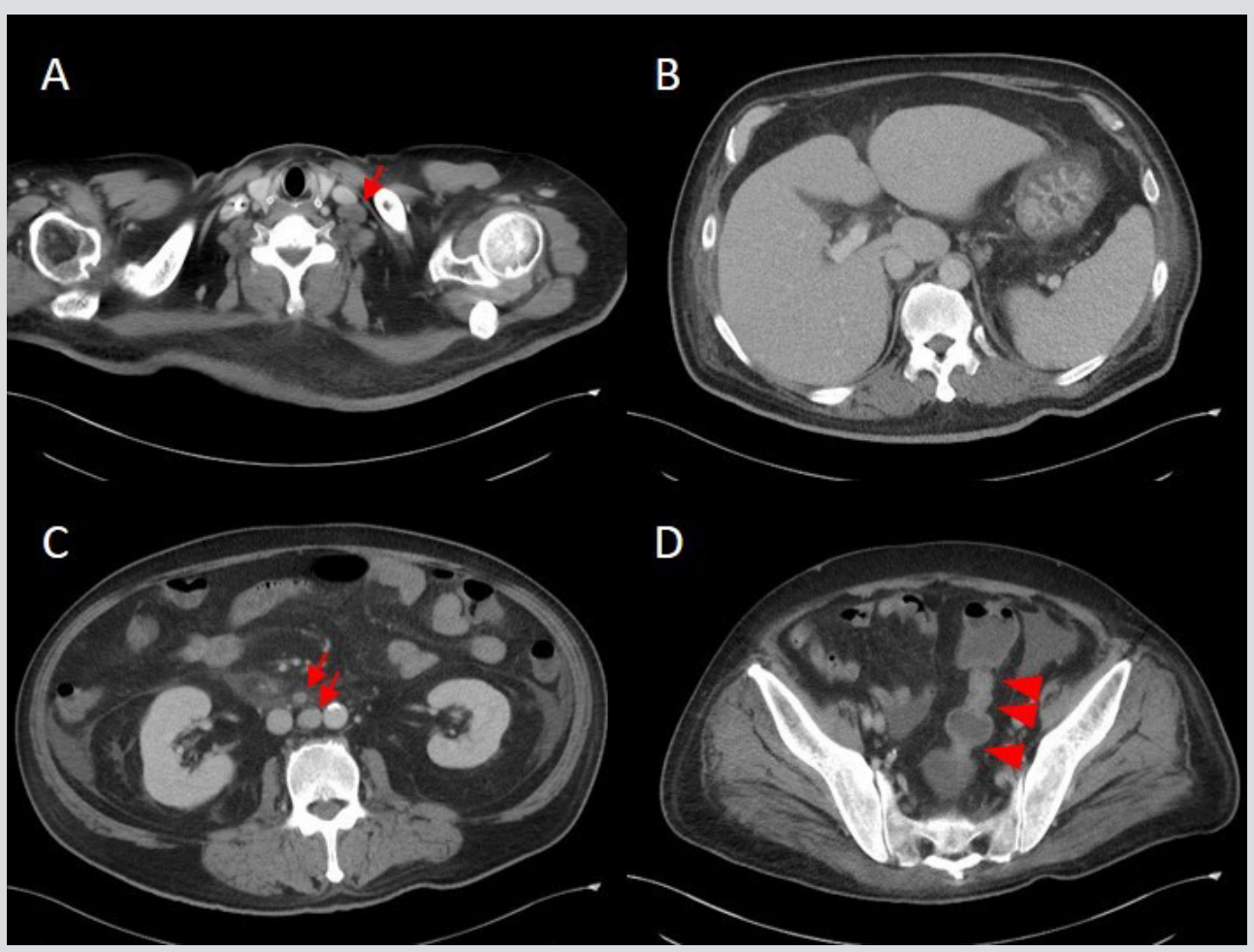

Figure 1. Contrast-enhanced chest and abdominal CT on day 6. (A) An enlarged lymph node on the left supraclavicular fossa (arrow). (B) Mild splenomegaly and slight thickening of the gastric wall. (C) Enlarged lymph nodes located on the paraabdominal aorta. (D) Partial narrowing of the sigmoid colon (arrow heads) 
Methods and Procedures

At this point, haemophagocytic lymphohistiocytosis (HLH) secondary to bone marrow involvement in malignant lymphoma, malignancy including gastric carcinoma, and colon carcinoma were suspected based on the pancytopenia and highly elevated serum ferritin. Infection was thought less likely as there was no evidence of it. Because the enlarged lymph nodes were too deep to sample under local anaesthesia, lymph node biopsy was deferred, and upper and lower gastrointestinal endoscopy was performed on days 12 and 13 , respectively, but did not reveal any specific findings of malignancy. Dry tap bone marrow aspiration was performed on the left iliac bone on day 14 , while biopsy showed haemophagocytosis, prominent fibrosis (Fig. 2A) and CD30-positive atypical cells (Fig. 2B). Repeat bone marrow biopsy on day 17 showed progression of the fibrosis.

Malignant lymphoma was still a probable diagnosis based on the highly elevated sIL-2R level (23,500 U/ml on day 13$)$ and the presence of CD30 positive atypical cells in the bone biopsy specimen. Consequently, biopsy of the supraclavicular lymph nodes was planned. Meanwhile, severe thrombocytopenia and renal dysfunction developed, with worsening of pleural effusion and ascites. The presence of myelofibrosis suggested the differential diagnosis of TAFRO syndrome, but the delayed onset of renal dysfunction and highly elevated sIL-2R level pointed against that diagnosis. Hypotension occurred before the results of the lymph node biopsy were received, and the patient was started on noradrenaline and steroid pulses.

On day 21, a left supraclavicular lymph node biopsy under general anaesthesia was performed. On day 27, the results of histology of the lymph node specimen revealed the presence of Hodgkin cells and Reed-Sternberg cells (Fig. 2C), confirming the diagnosis of stage IV Hodgkin's lymphoma complicated with myelofibrosis and haemophagocytic syndrome. Given the patient's condition, he was treated only with brentuximab vedotin and dacarbazine instead of the standard ABVD therapy. While waiting for the result of the biopsy, he developed multiple organ failure including severe acute liver failure (Table 1) and died on day 32.
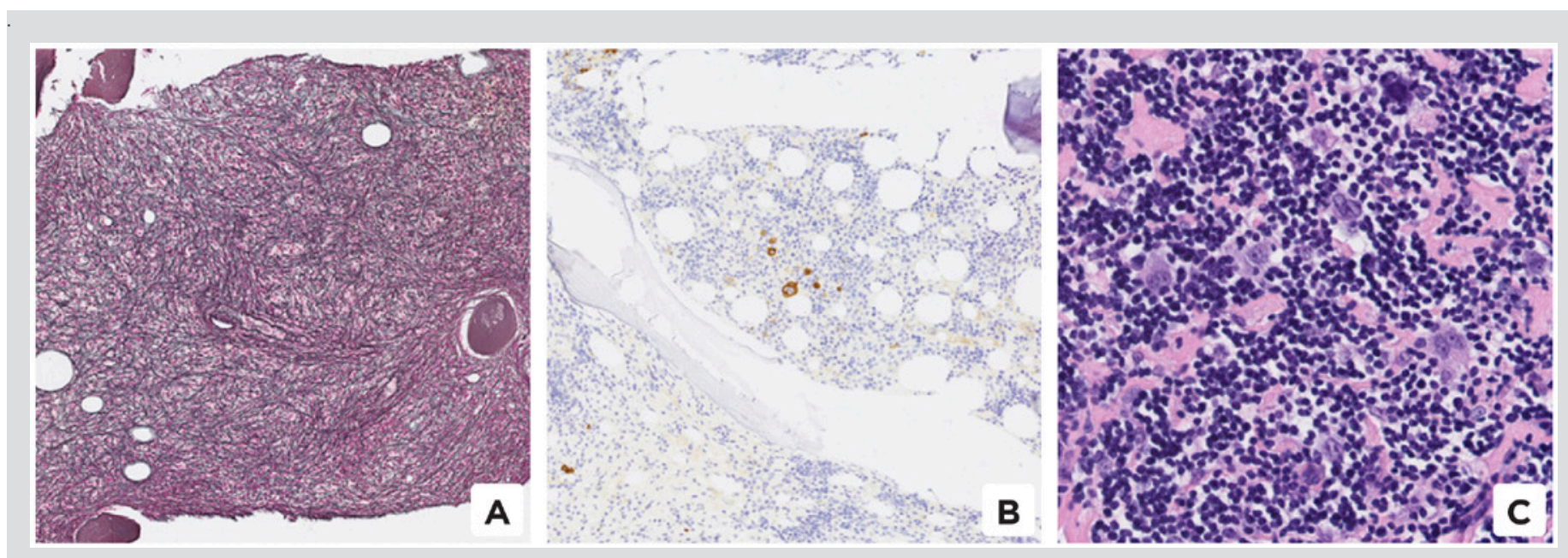

Figure 2. Pathological findings from bone marrow and lymph node biopsies. Bone marrow biopsy showing (A) prominent fibrosis and (B) some CD-30 positive atypical cells. Left supraclavicular lymph node biopsy showing (C) Hodgkin and Reed-Sternberg cells

\section{DISCUSSION}

This case illustrates the difficulty of making a correct and timely diagnosis of malignant lymphoma. The delayed diagnosis and the resulting clinical outcome were possibly associated with the delay in taking a lymph node biopsy. This was an atypical case of Hodgkin's lymphoma as HLH and myelofibrosis have rarely been reported in Hodgkin's lymphoma ${ }^{[1]}$. However, in retrospect, detailed clinical reasoning based on the evidence could have led the physicians to consider taking an earlier lymph node biopsy for confirmation of the diagnosis of Hodgkin's lymphoma.

This patient had lymph node enlargement in the left supraclavicular fossa. Although metastatic carcinomas were dominant (63\%) in these lymph nodes, around 9\% were lymphoid malignancies ${ }^{[2]}$. Most cases of secondary HLH due to malignancies are associated with haematological malignancies, and solid tumours such as gastric or colorectal carcinomas have been very rarely reported ${ }^{[3]}$. 
In this case, the lymph nodes were deeply located, such that biopsy with local anaesthesia could not be done. Therefore, rather than lymph node biopsy we chose to perform a bone biopsy from the left iliac bone, which showed prominent myelofibrosis. The finding of myelofibrosis misled the physicians to consider TAFRO syndrome as a differential diagnosis. However, there were some discrepancies between this case and previously reported cases of TAFRO syndrome. For instance, the sIL-2R level in this case was much higher than that seen in TAFRO syndrome. Indeed, it is noted in the proposed diagnostic criteria of TAFRO syndrome, that malignant lymphoma should be suspected in patients with a very high level of sIL-2R ${ }^{[4]}$. These findings might be helpful to discriminate lymphoma from TAFRO syndrome. Therefore, lymph node biopsy was still necessary before treatment for TAFRO syndrome in this case.

Another interesting point in this case is the clinical application of Sutton's law, where the health professional focusses on what seems to be most beneficial for the diagnosis. The law is named after the bank robber Willie Sutton, who reputedly replied to a reporter's inquiry as to why he robbed banks by saying "because that's where the money is". In this case, the supraclavicular lymph node was 'the bank', and therefore, the lymph node biopsy should have been performed at a very early stage in the diagnostic process. Compared to the risks of endoscopy or bone marrow biopsy (in general, below $0.1 \%$ ), the risk of mortality related to biopsy under general anaesthesia in severely ill patients (based on the P-POSSUM score ${ }^{[5]}$, our patient's predicted mortality risk was about 30-50\% soon after admission) can be very $\mathrm{high}^{[6-8]}$. Hence, it may be better to postpone taking a biopsy until the patient's clinical condition improves, although in this case, there was no guarantee that the patient would improve. Therefore, unnecessarily delaying a useful test may result in losing the chance of further testing, or increase the risk of doing a biopsy, especially if the patient's condition worsens. To that end, it is suggested that physicians should risk carrying out the most useful test for the diagnostic process even if it is invasive. And the decision to do the test should be made swiftly for timely diagnosis and treatment, and to minimize the risk of the test itself. The 'bank' may not be there indefinitely to help diagnosis.

Experienced physicians have an established intuitive thought process of rapidly recalling diagnoses. But intuitive thinking may be susceptible to bias. However, analytic diagnostic thinking can compensate for this shortcoming and complement intuitive diagnostic thinking.

This case underscores two reflections from the diagnostic medicine perspective. Physicians can reach the correct diagnosis by recalling a cluster of diseases that have a similar clinical expression to their intuitive diagnosis ${ }^{[9]}$. Moreover, in order to identify the correct differential diagnosis, physicians must understand the exact illness script of each disease and the differences in clinical findings among the differential diagnoses. The key to reaching the correct diagnosis as quickly as possible includes flexible use of the dual-process model and careful clarification of the clinical presentation of each differential diagnosis.

\section{REFERENCES}

1. Fu R, Yu H, Wu YH, Liu H, Shao ZH. Hodgkin's lymphoma associated with myelofibrosis: a case report. Oncol Lett 2015;10:1551-1554.

2. Nasuti JF, Mehrotra R, Gupta PK. Diagnostic value of fine-needle aspiration in supraclavicular lymphadenopathy: a study of 106 patients and review of literature. Diagn Cytopathol 2001;25:351-355

3. Ali M, Jain S, Wig N, Biswas A, Kumar A, Kumar K. Colonic adenocarcinoma presenting as hemophagocytic syndrome. Asian J Oncol 2017;3:92-95.

4. Masaki Y, Kawabata H, Takai K, Tsukamoto N, Fujimoto S, Ishigaki Y, et al. 2019 Updated diagnostic criteria and disease severity classification for TAFRO syndrome. Int $J$ Hematol 2020;111:155-158.

5. Prytherch DR, Whiteley MS, Higgins B, Weaver PC, Prout WG, Powell SJ. POSSUM and Portsmouth POSSUM for predicting mortality. Physiological and Operative Severity Score for the enUmeration of Mortality and morbidity. Br J Surg 1998;85:1217-1220.

6. Wolfsen HC, Hemminger LL, Achem SR, Loeb DS, Stark ME, Bouras EP, et al. Complications of endoscopy of the upper gastrointestinal tract: a single-center experience. Mayo Clin Proc 2004;79:1264-1267.

7. Bain BJ. Morbidity associated with bone marrow aspiration and trephine biopsy - a review of UK data for 2004. Haematologica 2006;91:1293-1294.

8. Reumkens A, Rondagh EJ, Bakker CM, Winkens B, Masclee AA, Sanduleanu S. Post-colonoscopy complications: a systematic review, time trends, and meta-analysis of population-based studies. Am J Gastroenterol 2016;111:1092-1101.

9. Shimizu T, Tokuda Y. Pivot and cluster strategy: a preventive measure against diagnostic errors. Int J Gen Med 2012;5:917-921. 